\title{
Symbol Error Rate Evaluation for OFDM Systems with MPSK Modulation
}

\author{
Yuhong Wang and Xiao-Ping Zhang \\ Department of Electrical and Computer Engineering, Ryerson University \\ 350 Victoria Street, Toronto, Ontario, Canada, M5B 2K3 \\ Email: xzhang@ee.ryerson.ca
}

\begin{abstract}
Orthogonal frequency division multiplexing (OFDM) is the most commonly used multiplexing method in multicarrier (MC) systems. In this paper, a new closedform formula for the symbol error rate (SER) is derived for generic OFDM systems with $M$-ary phase shift keying (MPSK) modulation and the optimal phase detector for each subchannel over a fading channel. This new formula can be used to evaluate both discrete Fourier transform (DFT)-based OFDM and discrete wavelet multitone (DWMT) systems. It can also be adopted as a criterion for OFDM system design. Monte-Carlo numerical simulations are performed to verify the theoretical SER analysis, and it is shown that the theoretical results from the presented new formula is consistent with simulation results.
\end{abstract}

\section{INTRODUCTION}

The orthogonal frequency division multiplexing (OFDM), as the most important class of multicarrier modulation (MCM), has been under intensive research and development in recent years, and $M$-ary phase shift keying (MPSK) has been adopted as an important modulation scheme for OFDM systems [1], [2]. A generally recognized advantage of OFDM is its robustness against various types of channel distortions, such as multipath propagation and narrowband interference [3], [4]. In OFDM modulation, subcarriers are mutually orthogonal such that the receiver can separate these subcarriers even if there is spectral overlapping among them. The filter bank formulation of the modulator and demodulator of a generic OFDM system is illustrated in Fig. 1.

In practical OFDM systems, the mutual orthogonality of subchannels is often destroyed by non-ideal channels. Therefore the transmitted symbols for a given

This work is supported in part by Canadian NSERC research grant RGPIN239031. subchannel may be distorted by co-subchannel symbols (intersymbol interference or ISI) and symbols of other subchannels (interchannel interference or ICI), as well as channel noise. The symbol error rate (SER) is an essential parameter for OFDM system performance evaluations. Due to the existence of ISI, ICI and channel noise in an OFDM system, the expression of SER cannot be obtained readily.

Recently some attempts have been made to analyze the error performance of discrete Fourier transform (DFT)-based systems such as in [5], [6]. The DFTbased OFDM system is only a special case of the OFDM where the DFT filter banks are used as modulators and demodulators. The error performance of generic OFDM systems, where general orthogonal modulation/demodulation filter banks are used, including discrete wavelet multitone (DWMT) and DFT-based OFDM, has not been systematically investigated.

The contribution of this paper lies in the systematic investigation of the error performance of a generic OFDM system, with DFT-based OFDM and DWMT as two realizations. Both interferences and additive Gaussian channel noise are considered in the analysis. By studying the constellations of received symbols and interferences for a given subchannel, and stochastic modeling the sum of ISI and ICI, the error performance of a given subchannel in a generic OFDM system is analyzed in the presence of additive Gaussian noise. Furthermore, by adopting the conventional optimal phase detector in each subchannel, we present a new closed-form SER expression for a generic OFDM system employing MPSK modulation. This new SER formula is verified with Monte-Carlo simulations by employing DFT-based OFDM systems and conventional DWMT systems. It is shown that the theoretical results from the presented new formula is consistent with simulation results. The 
presented new formula provides a viable tool for generic OFDM system design and performance evaluation.

\section{Symbol ERror Rate Evaluation FOr OFDM SYSTEMS WITH MPSK MODULATION}

\section{A. System Modeling}

The filter bank based modulator and demodulator, with $P$ subchannels, of a generic OFDM system are illustrated in Fig. 1. The modulation and demodulation filters of subchannel $p, 0 \leq p \leq P-1$, are denoted respectively as $f_{p}$ and $h_{p}$, which are of length $L=g K$, where $g$ is the overlapping factor which is a positive integer. The upsampling/downsampling factor is donated as $K$. The modulation filters $f_{p}, 0 \leq p \leq P-1$, should fulfill the orthogonality property [3], i.e., for $0 \leq p_{1}, p_{2} \leq P-1$

$$
\sum_{l=0}^{L-1} f_{p_{1}}(l) f_{p_{2}}^{*}(l-n K)=\delta(n) \delta\left(p_{1}-p_{2}\right) .
$$

Moreover, the modulation/demodulation filters satisfy the perfect reconstruction (PR) property defined as [7]

$$
\sum_{l=0}^{L-1} f_{p_{1}}(l) h_{p_{2}}(n K-l)=\delta(n) \delta\left(p_{1}-p_{2}\right) .
$$

The transmission channel $c(n)$ is modeled as a linear time invariant (LTI) FIR (finite impulse response) filter of length $L_{c}$, followed by a stationary zero-mean Gaussian noise source $e(n)$, which is independent of transmitted symbols $\left\{x_{p}(n), p=0,1, \cdots, P-1\right\}$. Note that if a time domain equalizer (TEQ) is employed preceding the filter bank demodulator, $c(n)$ can be considered as the overall transfer function resulting from the real communication channel and the TEQ.

For an OFDM system with MPSK modulation scheme, abbreviated as an MPSK-OFDM system, a conventional optimal phase detector is employed for each subchannel to calculate the phase of each received symbol and decode the received symbol with preset phase boundaries. The SER for a generic MPSK-OFDM system is derived in the following.

\section{B. Symbol Error Rate for Generic MPSK-OFDM Sys- tems}

In OFDM systems, at each time frame $n_{1}, P$ parallel symbols $\left\{x_{0}\left(n_{1}\right), x_{1}\left(n_{1}\right), \cdots, x_{P-1}\left(n_{1}\right)\right\}$ are transmitted, with each symbol modulated to one of the $P$ subchannels. The transmitted subchannel symbols in subchannel $p_{1}$ at frame $n_{1}, x_{p_{1}}\left(n_{1}\right)$, represent constellation points generated by a modulation scheme such as
MPSK. Corresponding to each transmitted constellation point, $x_{p_{1}}\left(n_{1}\right)$, there is a received symbol $y_{p_{1}}\left(n_{1}+d\right)$, where $d$ is the system delay. If only the delay involved in the convolution with the modulation filter and demodulation filter, both of length $L=g K$, is considered, then $d=g$. The following formulation gives the expression of $y_{p_{1}}\left(n_{1}+d\right)$.

Formulation 1: In an OFDM system shown in Fig. 1, suppose the channel impulse response is $c(n), n=$ $0,1, \cdots, L_{c}-1$, and the zero-mean additive channel Gaussian noise is $e(n)$, then for a given subchannel $p_{1}$, the received symbol $y_{p_{1}}\left(n_{1}+d\right)$, corresponding to the symbol $x_{p_{1}}\left(n_{1}\right)$ transmitted at time frame $n_{1}$, can be expressed as:

$$
y_{p_{1}}\left(n_{1}+d\right)=\bar{y}_{p_{1}}+\xi+\zeta
$$

where $\bar{y}_{p_{1}}$ is the contribution from symbol $x_{p_{1}}\left(n_{1}\right), \xi$ is the contribution from channel noise, and $\zeta$ is the interference item calculated by summing up all ICI and ISI items. Note that $x_{p_{1}}\left(n_{1}\right)$ is the only transmitted symbol contributing to $\bar{y}_{p_{1}}$, which can be expressed as

$$
\bar{y}_{p_{1}}=\alpha_{p_{1} p_{1}}\left(n_{1}\right) x_{p_{1}}\left(n_{1}\right) .
$$

The contribution from the channel noise, $\xi$, is calculated as

$$
\xi=\sum_{n=-\infty}^{\infty} e(n) h_{p_{1}}\left(n_{1} K-n\right),
$$

and the interference term $\zeta$ can be calculated as

$$
\begin{aligned}
\zeta= & \sum_{n=-\infty}^{\infty} \sum_{p \neq p_{1}, p=0}^{P-1} \alpha_{p p_{1}}(n) x_{p}(n) \\
& +\sum_{n \neq n_{1}, n=-\infty}^{\infty} \alpha_{p_{1} p_{1}}(n) x_{p_{1}}(n) .
\end{aligned}
$$

In (4) and (6), $\alpha_{p p_{1}}(n)$, for $0 \leq p, p_{1} \leq P-1$, is the scalar weight of the contribution from the transmitted symbol $x_{p}(n)$ to the received symbol $y_{p_{1}}\left(n_{1}+d\right)$, and it is calculated as:

$$
\alpha_{p p_{1}}(n)=\sum_{j=0}^{L_{c}-1} c(j) \sum_{l=0}^{L-1} f_{p}(l) h_{p_{1}}\left[\left(n_{1}-n\right) K-j-l\right],
$$

where $L_{c}$ is the length of channel impulse response $c(n)$.

The derivation details [8] of the above formulation are omitted due to the limit of space.

Remarks: When filter bank based modulator / demodulator and the communication channel are all linear and time invariant, the term $\bar{y}_{p_{1}}$ in (3) is deterministic 


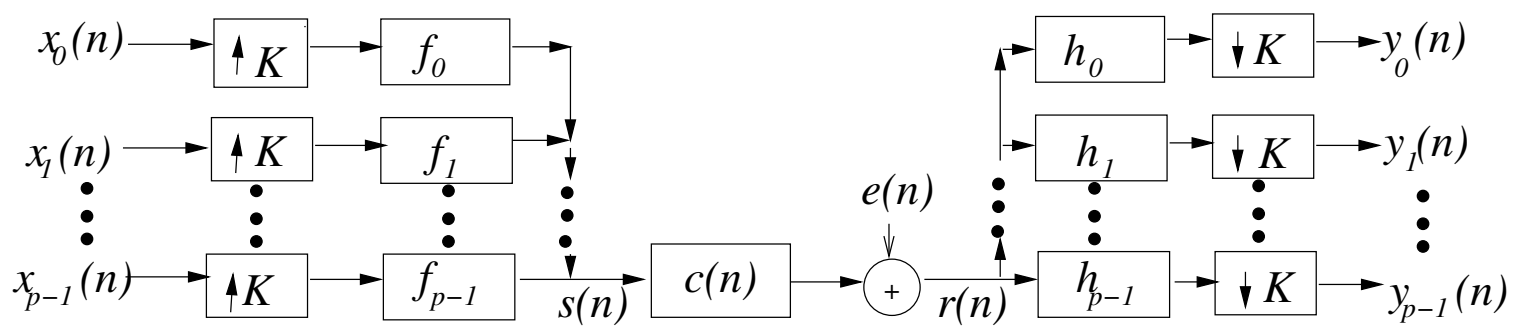

Fig. 1. Block diagram for filter bank based modulator and demodulator in OFDM systems

for a given transmitted symbol $x_{p_{1}}\left(n_{1}\right)$. According to (4), $\bar{y}_{p_{1}}$ is a weighted version of $x_{p_{1}}\left(n_{1}\right)$ and the weight $\alpha_{p_{1} p_{1}}\left(n_{1}\right)$ is a constant for a given subchannel $p_{1}$. Therefore in general, the constellation points of $x_{p_{1}}\left(n_{1}\right)$ and $\bar{y}_{p_{1}}$ form a one-to-one mapping. For each constellation point of $x_{p_{1}}\left(n_{1}\right)$, there is a corresponding constellation point $\bar{y}_{p_{1}}$, as calculated by (4). All the possible values of $\bar{y}_{p_{1}}$ form the set of constellation points at the receiving end of subchannel $p_{1}$. The noise item $\xi$ and interference item $\zeta$ are both random processes.

The following theorem can be proved [8] to provide a closed-form SER formula for MPSK modulation by calculating the probability density function (PDF) of $y_{p_{1}}\left(n_{1}+d\right)$ and setting the detection boundaries for optimal phase detector of each subchannel.

Theorem 1: When the transmitted symbols, $x_{p}(n), 0 \leq p \leq P-1$, are coded by the MPSK scheme with symbol energy $\epsilon_{x}$, and the optimal phase detector for each subchannel is adopted at the receiver end, the SER of the received symbol $y_{p_{1}}\left(n_{1}+d\right)$ at subchannel $p_{1}$, which corresponds to the transmitted symbol $x_{p_{1}}\left(n_{1}\right)$, can be determined as:

$$
P_{e, p_{1}}=\frac{1}{\pi} \int_{0}^{\frac{(M-1) \pi}{M}} \exp \left[-\frac{\gamma \sin ^{2}(\pi / M)}{\sin ^{2}(\phi)}\right] d \phi,
$$

where

$$
\gamma=\frac{\left|\alpha_{p_{1} p_{1}}\left(n_{1}\right)\right|^{2} \epsilon_{x}}{\left[\sum_{p, p \neq p_{1}} \sum_{n}\left|\alpha_{p p_{1}}(n)\right|^{2}+\sum_{n, n \neq n_{1}}\left|\alpha_{p_{1} p_{1}}(n)\right|^{2}\right] \epsilon_{x}+\sigma_{n}^{2}}
$$

is the signal to noise and interference ratio (SNIR), $\sigma_{n}^{2}$ is the variance of zero mean additive Gaussian noise $e(n)$, and $\alpha_{p p_{1}}(n)$ is defined in (7). The overall SER for the OFDM system, $P_{e}$, can be obtained by averaging $P_{p_{1}}$ over all subchannels,

$$
P_{e}=\frac{1}{P} \sum_{p_{1}=0}^{P-1} P_{e, p_{1}} .
$$

\section{SER Expression for Additive White Gaussian Noise (AWGN) Channels}

For an AWGN channel or a channel which have been perfectly equalized, $c(n)=\delta(n)$. The SER expression for a generic OFDM system can then be simplified. From (2) and (7),

$$
\alpha_{p p_{1}}(n)=\left\{\begin{array}{cc}
1 & p=p_{1} \text { and } n=n_{1} \\
0 & \text { otherwise }
\end{array}\right.
$$

i.e., the only symbol that has nonzero contribution to $y_{p_{1}}\left(n_{1}+d\right)$ is $x_{p_{1}}\left(n_{1}\right)$. Since $\sigma_{y}^{2}=\sigma_{n}^{2}$, the SNIR $\gamma$ in (9) becomes

$$
\gamma=\rho=\frac{\epsilon_{x}}{\sigma_{n}^{2}}
$$

which is the signal-to-noise ratio (SNR). From (10), the overall SER, $P_{e}$, is

$$
P_{e}=P_{e, p_{1}} .
$$

By substituting (11) into (8), we get the SER for an OFDM system which is identical to the SER formula for single carrier systems over an AWGN channel. With the increase of SNR, SER will decrease quickly. We can also see from (13) that for an AWGN channel, the number of subchannel, $P$, has no effect on average system error performance.

Fig. 2 shows the SER results for both single carrier modulation and the conventional DWMT modulation, with different numbers of subchannels, over an AWGN channel. The modulation scheme adopted is binary phase shift keying (BPSK).

\section{SER Expression for Fading Channels}

For fading channels, we rewrite $\gamma$ in (9) in an alternative expression as

$$
\gamma=\frac{\left|\alpha_{p_{1} p_{1}}\left(n_{1}\right)\right|^{2} \rho}{\left[\sum_{p, p \neq p_{1}} \sum_{n}\left|\alpha_{p p_{1}}(n)\right|^{2}+\sum_{n, n \neq n_{1}}\left|\alpha_{p_{1} p_{1}}(n)\right|^{2}\right] \rho+1} .
$$




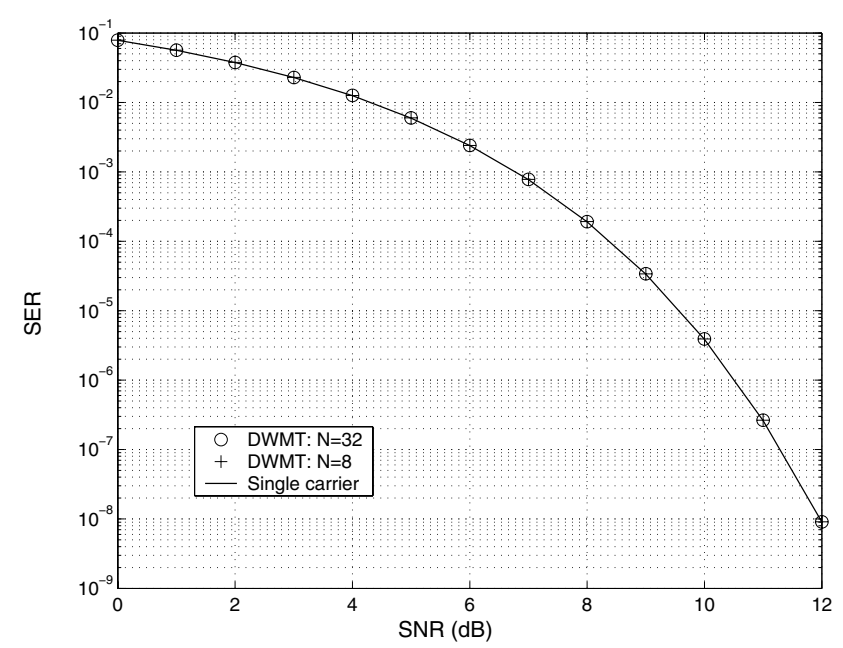

Fig. 2. Error performance of single carrier modulation and the conventional DWMT $(g=2)$ modulation with BPSK.

It can be seen that when $\operatorname{SNR} \rho$ is much larger than 1 $(0 \mathrm{~dB}), P_{e, p_{1}}$ is dominated by the weights $\alpha_{p p_{1}}(n)$, and can be approximated as

$$
\gamma \approx \frac{\left|\alpha_{p_{1} p_{1}}\left(n_{1}\right)\right|^{2}}{\sum_{p, p \neq p_{1}} \sum_{n}\left|\alpha_{p p_{1}}(n)\right|^{2}+\sum_{n, n \neq n_{1}}\left|\alpha_{p_{1} p_{1}}(n)\right|^{2}} .
$$

In this case, the SER performance will not change much with the increase of SNR $\rho$. Fig. 3 shows the error performance of a DWMT system, with overlapping factor $g=2$, over a sample fading channel.

For DFT-based OFDM systems, from (7), the inclusion of zero-padded cyclic prefix does not change the value of numerator in (15), but the value of denominator will decrease because of the increase of the upsampling/downsampling factor $K$. Therefore, OFDM systems with cyclic prefix will demonstrate superior error performance than OFDM systems without cyclic prefix.

Expressions of (8) and (10) provide a systematic approach to calculate the SER of a generic OFDM system, which fulfills the orthogonality and PR conditions in (1) and (2). Moreover, the SER formula can be used as objective functions in the design of modulation and demodulation filter banks for an OFDM system to achieve better error performance.

\section{Simulations}

To verify our theoretical analysis of error performance of a generic OFDM system presented in Theorem

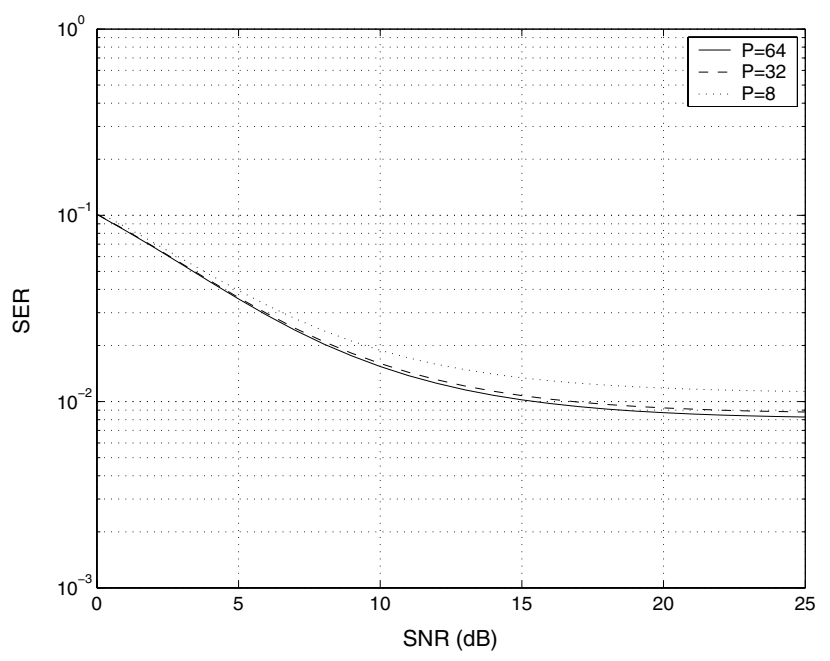

Fig. 3. Error performance of conventional DWMT systems $(g=2)$ over channel $c(n)=\left[1,0.5 e^{j \pi / 6}, 0.3 e^{-j \pi / 3}, 0.2 e^{j \pi / 2}, 0.1\right]$.

1, Monte-Carlo numerical simulations are performed with three types of OFDM systems, namely, DFTbased OFDM with zero-padded cyclic prefix, the DFTbased OFDM without cyclic prefix and the conventional DWMT systems [3] with overlapping factor $g=2$, of which the modulation filters are generated with prototype filter

$$
w(l)=\sin \left[\frac{\pi}{2 P}\left(l+\frac{1}{2}\right)\right],
$$

for $l=0,1, \cdots, 2 P-1$.

Transmitted data stream $x_{p}(n), p=0,1, \cdots, P-1$, are generated using the QPSK scheme. The composite data sequence before entering the channel, $s(n)$, is obtained as illustrated in Fig. 1. AWGN noise with a particular SNR is added to obtain distorted data sequence $r(n)$. Then $r(n)$ is passed through the demodulation filter of each subchannel, and the result signals are downsampled by factor $K$. The optimal phase detector is employed in each subchannel. The detected symbols are compared with the original data and errors are counted to obtain SER for a particular SNR.

Figs. 4 and 5 show the results of these OFDM systems, with $P=32$ subchannels, over two sample multipath fading channels with impulse response $c_{1}(n)=\left[1,0.5 e^{j \pi / 6}\right]$ and $c_{2}(n)=\left[1,0.5 e^{j \pi / 6}, 0.3 e^{-j \pi / 3}, 0.2 e^{j \pi / 2}, 0.2,0.1\right]$ for QPSK symbols. The simulation results demonstrate consistence with the corresponding theoretical analysis. 
As expected, DFT-based OFDM system with cyclic prefix has superior SER over the other two OFDM systems, with the cost of transmission efficiency due to zero padding. The DWMT has the poorest SER since both channels have no narrow band interference and hence the ISI has more significant effect on SER than ICI (which DWMT is designed to minimize). Moreover, since the filters in DWMT are of longer length, the dispersive channel could distort more symbols and produce more ISI.

\section{CONCLUSiOnS}

In the past, the error performance of a generic OFDM system, in which the modulation filters form a set of generic orthonormal basis, has not been systematically investigated. In this paper, a new closed-form SER formula is presented for a generic OFDM system with MPSK modulation and optimal phase detection in each subchannel, based on the constellation points of received symbols and statistical analysis of ISI and ICI. This formula can be used to evaluate both DFTbased OFDM and DWMT systems. Simulations are performed with DFT-based OFDM systems with and without zero-padded cyclic prefix, and the DWMT system with overlapping factor $g=2$. The results show the consistency between theoretical SER analysis and numerical simulations. The presented new formula provides an important tool to analyze the performance of a generic OFDM system and it provides a realistic way to formulate an objective function to design an OFDM system [8].

\section{REFERENCES}

[1] V. Mignone and A. Morello, "CD3-OFDM: a novel demodulation scheme for fixed and mobile receivers," IEEE Trans. on Communications, vol. 44, no. 9, pp. 1144-1151, Sept. 1996.

[2] C. Liu, "The effect of nonlinearity on a QPSK-OFDM-QAM signal," IEEE Trans. on Consumer Electronics, vol. 43, no. 3, pp. 443-447, Aug. 1997.

[3] S. D. Sandberg and M. A. Tzannes, "Overlapped discrete multitone modulation for high speed copper wire communications," IEEE Journal on Selected Areas in Commu., vol. 13, no. 9, pp. 1571-1585, Dec. 1995.

[4] A. N. Akansu and X. Lin, "A comparative performance evaluation of DMT(OFDM) and DWMT(DSBMT) based DSL communications systems for single and multitone interference," in proc. ICASSP, vol. 6, pp. 3269-3272, May 1998.

[5] S. Lei and V. Lau, "Performance analysis of adaptive interleaving for OFDM systems," IEEE Trans. on. Vehicular Tech., vol. 51, no. 3, pp. 435-444, May 2002.
[6] T. Tjhung, X. Wang, and C.S. Ng, "Error performance evaluation of the MDPSK-DMT systems in AWGN and impulse noise," IEEE Trans. on Consumer Electronics, vol. 46, no. 1, pp. 131-136, Feb. 2000.

[7] P. P. Vaidyanathan, Multirate Systems and Filter Banks, Englewood Cliffs, NJ: Prentice Hall, 1993.

[8] Y. Wang and X.-P. Zhang, "Symbol error rate evaluation and filter bank design for OFDM systems with MPSK modulation," submitted for journal publication, 2003.

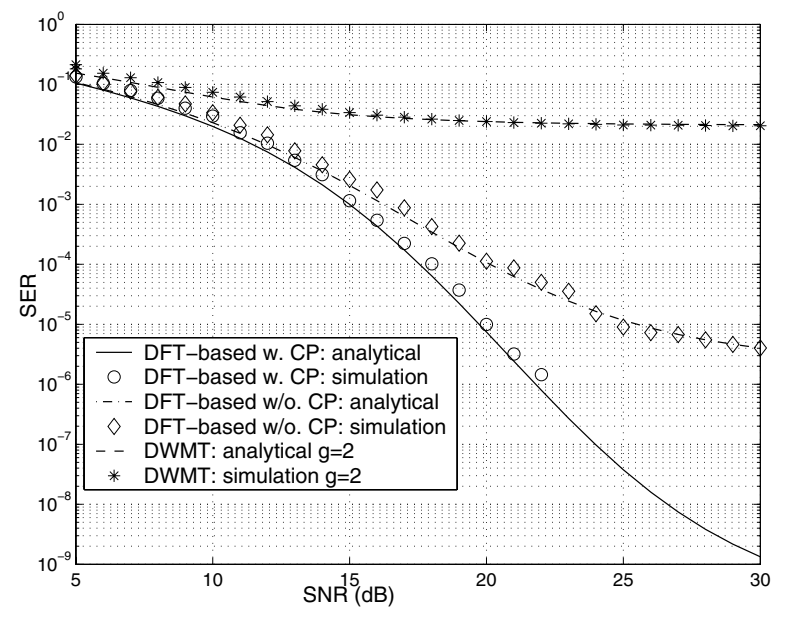

Fig. 4. Comparison of the analytical and simulated performance of OFDM systems with QPSK coding over channel $c_{1}(n)(P=32)$.

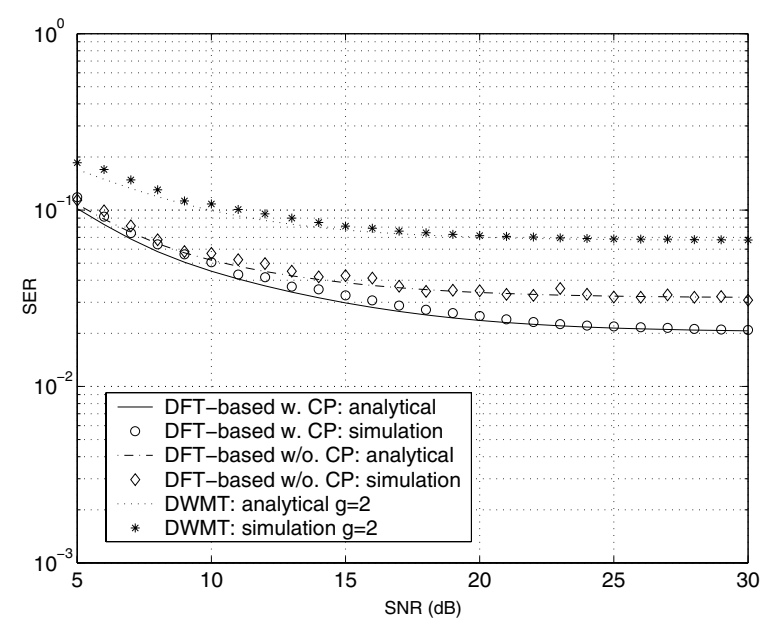

Fig. 5. Comparison of the analytical and simulated performance of OFDM systems with QPSK coding over channel $c_{2}(n)(P=32)$. 